\title{
Microbiology of wound infection after caesarean section in a Jordanian hospital
}

N.M. Kaplan, ${ }^{1}$ A.A. Smadi, ${ }^{2}$ M.I. Al-Taani ${ }^{3}$ and M.A. El-Qudah ${ }^{3}$

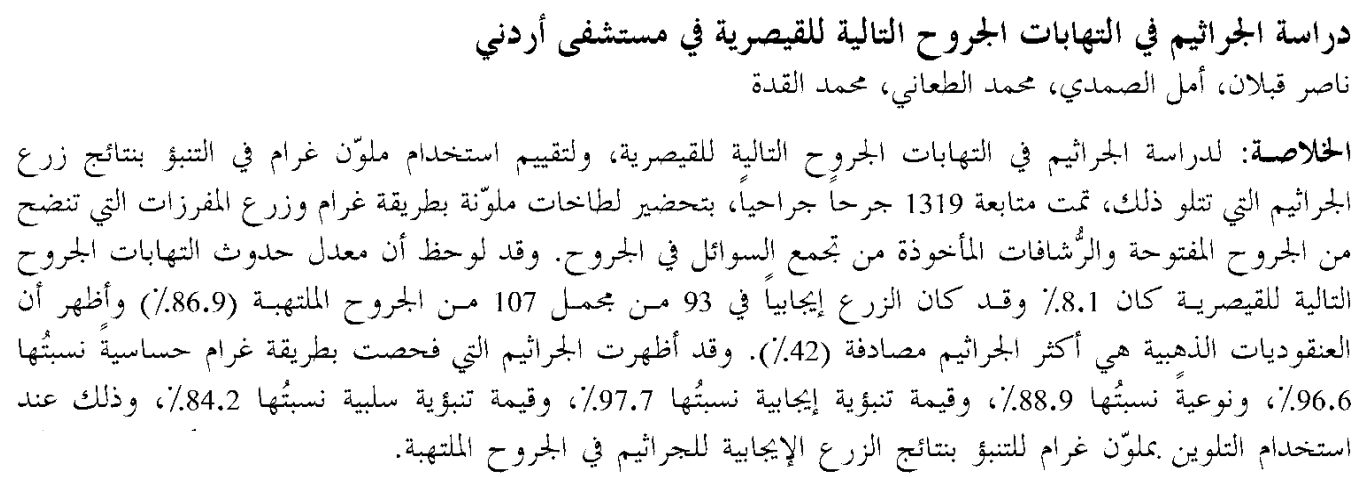

ABSTRACT To determine the microbiology of wound infection following caesarean section and to evaluate the use of Gram stain for the predicton of subsequent microbiological culture results, 1319 surgical wounds were followed up. We did Gram stains and cultures on exudates from open wounds and on aspirates if the wounds had demonstrable fluid collection. Incidence of post-caesarean wound infection was $8.1 \%$. Ninetythree (86.9\%) of 107 infected wounds were culture positive, with Staphylococcus aureus the most frequently found organism (42\%). Organisms seen by Gram stain yielded a sensitivity of $96.6 \%$, specificity of $88.9 \%$, positive predictive value of $97.7 \%$ and negative predictive value of $84.2 \%$ when used to predict positive culture results for bacterial wound infection.

Microbiologie de l'infection de la plaie après césarienne dans un hôpital jordanien

RESUME Afin de déterminer la microbiologie de l'infection de la plaie après une césarienne et d'évaluer l'utilisation de la coloration de Gram pour prévoir les résultats des cultures microbiologiques ultérieures, 1319 plaies chirurgicales ont fait l'objet d'un suivi. Nous avons procédé à une coloration de Gram et à des cultures sur des exsudats de plaies ouvertes et des échantillons prélevés par aspiration si la plaie avait une accumulation de fluides manifeste. L'incidence de l'infection de la plaie après césarienne s'élevait à $8,1 \%$. Quatre-vingt-treize (86,9\%) des 107 plaies infectées avaient des cultures positives, Staphylococcus aureus étant le micro-organisme le plus fréquemment trouvé (42\%). Les micro-organismes mis en évidence par coloration de Gram ont donné une sensibilité de $96,6 \%$, une spécificité de 88,9\%, une valeur prédictive positive de $97,7 \%$ et une valeur prédictive négative de $84,2 \%$ lorsqu'ils étaient utilisés pour prévoir les résultats de culture positifs pour les infections bactériennes des plaies.

${ }^{1}$ Department of Pathology, ${ }^{2}$ Department of Radiology; ${ }^{3}$ Department of Gynaecology and Obstetrics, Queen Alia Military Hospital, Amman, Jordan.

Received: 31/07/02; accepted: 26/02/03 


\section{Introduction}

Wound infection after caesarean delivery occurs in $2 \%-16 \%$ of patients, depending on factors such as antibiotic prophylaxis, length of labour, duration of rupture of membranes, duration of internal monitoring, and number of vaginal examinations $[1,2]$. In one case-control study conducted in a university hospital population, it was reported that $89 \%$ of 57 post-caesarean wound infections were culture-positive [3].

The Gram stain has been found to be highly specific but less sensitive in the prediction of post-caesarean endomyometritis [4] and in the early detection of significant burn wound microbial growth [5]. In these studies, infection was defined as microbiologic recovery of pathogenic organisms by culture.

The isolation of genital mycoplasmas from post-caesarean wound infections has also been reported, however pathogenicity in this setting was not precisely known. The Gram stain findings consistently showed predominantly white blood cells and no organisms [6,7].

We conducted this study to define the prevalence of pathogenic organisms in post-caesarean wound infection in our hospital and to evaluate the use of Gram stain to predict subsequent microbiological culture results. Cultures were not done for Mycoplasma hominis or Ureaplasma urealyticum (no special facilities available). The literature was also reviewed for the predisposing factors and preventive measures of wound infection following caesarean section.

\section{Methods}

We followed prospectively a total of 1319 women who underwent caesarean section at the Department of Obstetrics and Gynaecology, Queen Alia Military Hospita in the period between 1 October 1998 and 31 January 2002. The hospital has a fullyequipped, central sterile supply department and a regularly updated disinfection policy. The basic universal management of caesarean section was followed and all patients received $1 \mathrm{~g}$ of cephalothin sodium (Keflin) intravenously as prophylaxis at the time of umbilical cord clamping. A wound infection was identified by the presence of purulent discharge from the incision with erythematous cellulitis, induration or pain, and demonstrable fluid collection noted on ultrasound. Women with stitch abscesses, haematomas and seromas, or those developing infection after hospital discharge, were not included in this study.

Purulent exudates were obtained from the open discharging wounds with a sterile cotton swab. Aspirates were obtained by preparing the wound area with alcohol, inserting a sterile needle through the healing incision and aspirating fluid into a sterile syringe. Culturing was done within 1 hour using standard bacteriological inoculation techniques. Blood, chocolate (Diagnolab, Barcelona, Spain) and MacConkey (MAST Diagnostics, Merseyside, United Kingdom) agars were used to isolate Gram-positive and Gram-negative aerobic microorganisms. Schaedler agar (BBL Microbiology Systems, Cockeysville, Maryland, United States of America) was used for the isolation of anaerobes. The aerobic plates were read within 24-48 hours and the anaerobic plates at 48 and 72 hours. The anaerobic plates were kept 1 week before a final negative result was confirmed. Quantitative bacteriology was not performed. Any growth was subsequently identified by standard microbiological methods. Gram stains were also performed and recorded at the time of culturing. Primary culturing and

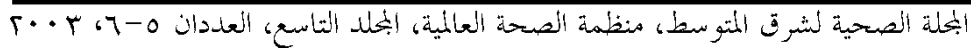


Gram staining of specimens were done by Medical Laboratory Scientific Officer Grade 1. Microscopic examination of Gram-stained slides and subsequent identification of bacterial isolates were done by an experienced senior microbiologist. The Gram stain results were defined as follows: a positive Gram stain requires that organisms with or without appreciable numbers of white blood cells were seen under oil immersion light microscopy; a negative Gram stain requires that no organisms were seen under oil immersion light microscopy.

The Gram stain results were studied in comparison with isolation of viable organisms in cultures. Sensitivity, specificity, and predictive values were calculated by standard formulae. A true positive was defined as a positive smear from a wound from which an organism was subsequently cultured within 48 hours. A false positive had a positive smear but a negative culture within 48 hours. A false negative had a negative smear but a positive culture within 48 hours of incubation. Fisher exact test was applied as a test of significance.

\section{Results}

Of 12083 women delivered during the study period; 1319 (10.9\%) had caesarean section. Of these, 107 (8.1\%) developed an abdominal incision infection during hospitalization, 93 of which (86.9\%) were classed as positive. A total of 112 organisms were isolated. There were 47 (42\%) Staphylococcus aureus, 31 (27.7\%) Escherichia coli, 23 (20.5\%) Klebsiella sp., 6 (5.3\%) Pseudomonas sp., 3 (2.7\%) Enterococcus sp. and 2 (1.8\%) anaerobes.

The wound was open in 87 (81\%) of the 107 cases. Eighty-three of the 87 (95.4\%) cultures from open wounds were positive.
Gram stains of the exudates and aspirates were used to predict subsequent microbiological culture results (Table 1). Organisms examined by Gram stain yielded a sensitivity of $96.6 \%$, specificity of $88.9 \%$, positive predictive value of $97.7 \%$ and negative predictive value of $84.2 \%$ when used to predict positive culture results for bacterial wound infection. The difference between positive and negative Gram staining for prediction of subsequent culture results was statistically significant $(P<0.0001)$ by Fisher exact test.

\section{Discussion}

Wound infection is a common surgical complication, often requiring a prolonged hospital stay and leading to increased costs. It represents the most common serious complication of caesarean section. There are at least two mechanisms responsible for the development of post-caesarean wound infection: first, increased amniotic fluid and wound colonization by cervicovaginal flora due to prolonged rupture of membranes, and second, increased exogenous bacterial contamination by skin flora due to breaks in sterile technique, often accompanying difficult or emergency surgery $[3,8]$.

Table 1 Results of Gram stain and culture of the 107 wound samples

\begin{tabular}{lccr}
\hline Gram stain & $\begin{array}{c}\text { Positive } \\
\text { culture }\end{array}$ & $\begin{array}{c}\text { Negative } \\
\text { culture }\end{array}$ & Total \\
\hline $\begin{array}{l}\text { Positive Gram } \\
\quad \text { stain }\end{array}$ & 86 & 2 & 88 \\
$\begin{array}{l}\text { Negative Gram } \\
\quad \text { stain }\end{array}$ & 3 & 16 & 19 \\
Total & 89 & 18 & 107 \\
\hline
\end{tabular}

$\mathrm{P}<0.0001$ by Fisher exact test. 
The commonest causative organism of post-caesarean section wound infection in our sample was $S$. aureus. One of the major problems facing the laboratory is distinguishing clinically significant, pathogenic strains of coagulase-negative staphylococci from contaminant strains [9]. The importance of coagulase-negative staphylococci is increasing due to the increase in the use of transient or permanent medical devices, such as intravascular catheters and prosthetic devices in seriously ill and immunocompromised patients. All coagulase-negative staphylococci in our study were isolated from open wounds and regarded as skin contaminants. There were only 2 anaerobic organisms isolated.

In all, 10 closed and 4 open wound cultures were negative and did not grow viable organisms. This might be attributed to difficult-to-grow fastidious organisms, inappropriate processing of specimens in the laboratory or the administration of antibiotics prior to specimen collection.

False positive Gram stain results could be due to either stained cotton swab fibres or stain deposits or crystals. False negative Gram stain results could be due to low numbers of organisms or inadequate screening of the smear. Gram stain proved to be simple, rapid, cheap and of acceptable predictive value.

Careful examination of Gram-stained slides is required to determine Gram-stain affinity, morphology and arrangement of the organisms and consequently guide the early choice of the appropriate antimicrobial agent. However it has to be emphasized that Gram stain should not be a substitute for culture.

The reported rate of wound infection after caesarean section ranges widely, largely because of different risk factors among diverse patient populations. In some studies, mean rate of wound infection after caesarean section was found to be $10 \%$ among women not receiving prophylactic antibiotics $[10,11]$. The incidence of postcaesarean section wound infection was $4.5 \%$ in a tertiary hospital in Saudi Arabia [12], however the rate in our sample was $8.1 \%$. Underestimation of wound infection rates has always been a concern as some hospitals send comparatively few swabs to the laboratory for examination, consequently any measure of infection that depends on routinely analysed swabs is likely to underestimate the actual level [13]. Similarly, wound infections may present later after discharge from hospital, as women who have a caesarean section usually have a relatively short stay. Hence, without follow-up in the community, underestimation may exist. [14-16], and one study reported that $36 \%$ of post-caesarean section wound infections were diagnosed following the patients' discharge from hospital [15]. Therefore, it is strongly recommended that data on post-discharge surveillance should always be included to realistically estimate the true rates of post-caesarean section wound infection and to allow the implementation of adequate preventive measures.

The incidence of post-caesarean wound infection has been found to be higher following emergency rather than elective caesarean section $[8,17,18]$, in general ward rather than private ward cases [19], in clinic rather than private patients [18] and in patients from lower rather than higher socioeconomic groups $[15,20]$.

Various risk factors have been assessed in relation to post-caesarean surgical site infection $[21,22]$. Prolonged rupture of membranes $[8,19]$, multiple pelvic examinations $[18,19]$, duration of operation, vertical skin incision, category of surgeon [18], maternal weight, obesity and thickness of subcutaneous tissue at the surgery

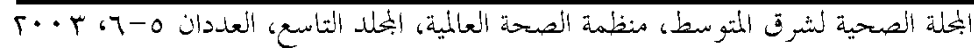


site $[15,20]$ and anaemia $[19,22]$ have been identified as statistically significant factors associated with a high risk of post-caesarean wound infection.

Antibiotic prophylaxis has been found to be the most significant protective factor in reducing both the rate of post-caesarean section wound infection $[15,23]$ and costs [24]. Most clinical trials have shown no significant difference in the efficacy of various antibiotic regimens [25]. However, antibiotic prophylaxis will not prevent infection if poor surgical techniques have been employed, and will result in the selection of resistant bacteria $[26,27]$. Postcaesarean wound infection caused by enterococci have been significantly associated with the use of cephalosporin prophylaxis [28]. Therefore, anxieties about antimicrobial toxicity (including allergic reactions), the potential to cause an increase in hospital-acquired infection with resistant organisms and the possible masking of early infection in the neonate have suggested that the overall risk-benefit ratio and cost-effectiveness may not be favourable.

A significant reduction in the rate and severity of postoperative endometritis after caesarean section has been reported in association with the application of strict preoperative hygienic routines. However, no similar reduction was found concerning wound infections [29]. The redisinfection of the skin around the caesarean incision before skin closure has been reported to reduce the incidence of postoperative wound infection [30], however no benefit from the use of adhesive plastic drapes could be demonstrated [30,31]. It has also been reported that antibiotic irrigation is safe, showing no noted adverse effects, and is an effective method in reducing post-caesarean section infectious morbidity and wound infections [32].

In a controlled clinical trial to study the treatment of postoperative wound infections following caesarean section or total abdominal hysterectomy, the topical application of crude undiluted honey was associated with faster eradication of bacterial infections, shorter periods of concomitant antibiotic use and hospital stay, acceleration of wound healing, prevention of wound dehiscence with the consequent need for re-suturing, and finally minimal scar formation [33].

\section{References}

1. Olson M, O'Connor M, Schwartz ML. Surgical wound infections: A 5-year prospective study of 20,193 wounds at Minneapolis VA Medical Center. Annals of surgery, 1984, 199:253-9.

2. Gibbs RS, Listwa HM, Read JA. The effect of internal fetal monitoring on maternal infection following caesarean section. Obstetrics and gynecology, 1976, 48:653-8.

3. Emmons SL et al. Development of wound infections among women under- going caesarean section. Obstetrics and gynecology, 1988, 72:559-64.

4. Duff $P$ et al. Correlation of laboratory and clinical criteria in the prediction of postcaesarean endomyometritis. Obstetrics and gynecology, 1984, 63:781-6.

5. Taddonio TE et al. Rapid quantification of bacterial and fungal growth in burn wounds: Biopsy homogenate Gram stain versus microbial culture results. Burns, including thermal injury, 1988, 14:1804. 
6. Maccato M, Faro S, Summers K. Wound infections after caesarean section with Mycoplasma hominis and Ureaplasma urealyticum. Diagnostic microbiology and infectious disease, 1990, 13:363-5.

7. Roberts $\mathrm{S}$ et al. The microbiology of postcesarean wound morbidity. Obstetrics and gynecology, 1993, 81:383-6.

8. Martens MG et al. Development of wound infection or separation after cesarean delivery. Prospective evaluation of 2,431 cases. Journal of reproductive medicine, 1995, 40:171-5.

9. Kleeman KT, Bannerman TL, Kloos WE. Species distribution of coagualse-negative staphylococcal isolates at a community hospital and implications for selection of staphylococcal identification procedures. Journal of clinical microbiology, 1993, 31:1318-21.

10. Gibbs RS, Blanco JD, St Clair PJ. A case-control study of wound abscess after cesarean delivery. Obstetrics and gynecology, 1983, 62:498-501.

11. Swartz WH, Grolle K, Corson SL. The use of prophylactic antibiotics in caesarean section, a review of the literature. Journal of reproductive medicine, 1981, 26: 595-609.

12. Habib FA. Incidence of post-caesarean section wound infection in a tertiary hospital, Riyadh, Saudi Arabia. Saudi medical journal, 2002, 23:1059-63.

13. Thompson JR, Hutton RM, Moir-Bussy BR. Estimating the infection rate in mothers following caesarean section. Journal of hospital infection, 1987, 10:138-44.

14. Couto RC et al. Post-discharge surveillance and infection rates in obstetric patients. International journal of gynecology and obstetrics, 1998, 61: 227-31.

15. Beattie et al. Risk factors for wound infection following caesarean section. Australian and New Zealand journal of obstetrics and gynaecology, 1994, 34: 398-402.

16. Parrott $T$ et al. Infection following caesarean section. Journal of hospital infection, 1989, 13:349-54.

17. Hillan EM. Postoperative morbidity following caesarean delivery. Journal of advanced nursing, 1995, 22:1035-42.

18. Webster J. Post-caesarean wound infection: a review of the risk factors. Australian and New Zealand journal of obstetrics and gynaecology, 1988, 28 : 201-7.

19. Nanda S, Rathee S. Postcaesarean wound infection: a review of risk factors. Journal of the Indian Medical Association, 1992, 90:66-7.

20. Vermillion ST et al. Wound infection after Caesarean: effect of subcutaneous tissue thickness. Obstetrics and gynaecology, 2000, 95:923-6.

21. Tran TS et al. Risk factors for postceasarean surgical site infection. Obstetrics and gynaecology, 2000, 95: 367-71.

22. Litta $P$ et al. Risk factors for complicating infections after caesarean section. Clinical and experimental obstetrics and gynecology, 1995, 22:71-5.

23. Normand MC, Damato EG. Postcaesarean infection. Journal of obstetric, gynecologic, and neonatal nursing, 2001, 30:642-8.

24. Mugford M, Kingston J, Chalmers I. Reducing the incidence of infection after caesarean section: implications of prophylaxis with antibiotics for hospital resources. British medical journal, 1989, 299:1003-6.

25. Fernandez H. Antibioprophylaxie dans les cesariennes et les interruptions volontaires de grossesse. [Antibiotic prophylaxis in caesarean section and voluntary termination in pregnancy.]

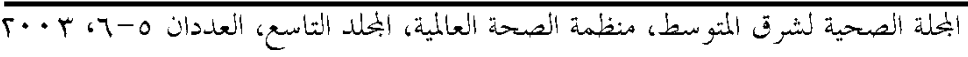


Annales francaises d'anesthesie et de reanimation, 1994, 13 (suppl.):128-34.

26. Faro S. Antibiotic prophylaxis. Obstetrics and gynecology clinics of North America, 1989, 16:279-89.

27. Faro S. Infectious disease relations to caesarean section. Obstetrics and gynecology clinics of North America, 1988, 15:685-95.

28. Walmer D, Walmer KR, Gibbs RS. Enterococci in post-cesarean endometritis. Obstetrics and gynaecology, 1988, 71: 159-61.

29. Hagglund L, Christensen KK, Christensen P. Effect of a strict preoperative hygienic routine on the rate of infections following caesarean section. European journal of obstetrics, gynecology, and reproductive biology, 1986, 23:187-94.

30. Cordtz $T$ et al. The effect of incisional plastic drapes and redisinfection of op- eration site on wound infection following caesarean section. Journal of hospital infection, 1989, 13:267-72.

31. Ward HR et al. Do plastic adhesive drapes prevent post caesarean wound infection? Journal of hospital infection, 2001, 47:230-4.

32. Levin DK, Gorchels C, Andersen R. Reduction of post-cesarean section infectious morbidity by means of antibiotic irrigation. American journal of obstetrics and gynecology, 1983, 146:273-7.

33. Al-Waili NS, Saloom KY. Effects of topical honey on post-operative wound infections due to Gram positive and Gram negative bacteria following caesarean sections and hysterectomies. European journal of medical research, 1999, 4: 126-30. 